\title{
Aggregative stability of fungicidal nanomodifier based on zinc hydrosilicates
}

\author{
Anna Grishina ${ }^{1, *}$ and Evgeniy Korolev ${ }^{1}$ \\ ${ }^{1}$ Moscow State University of Civil Engineering, Nanomaterials and Nanotechnology Research and \\ Educational Center, Yaroslavskoye hw. 26, 129337, Moscow, Russian Federation
}

\begin{abstract}
Currently, there is a strong need of high performance multi functional materials in high-rise construction. Obviously, such materials should be characterized by high strength; but for interior rooms biosafety is important as well. The promising direction to obtain both high strength and maintain biosafety in buildings and structures is to manage the structure of mineral binders by means of fungicidal nanomodifier based on zinc hydrosilicates. In the present work the aggregative stability of colloidal solutions of zinc hydrosilicates after one year of storage was studied. It has been established that the concentration of iron (III) hydroxide used to prepare the precursor of zinc hydrosilicates has a significant effect on the long-term aggregative stability: as the concentration of iron (III) hydroxide increases, the resistance of the fungicidal nanomodifier increases. It was found that, despite the minimal concentration of nano-sized zinc hydrosilicates $(0.028 \%)$, the colloidal solution possesses a low long-term aggregative stability; while in the initial period (not less than 14 days) the colloidal solution of the nanomodifier is aggregatively stable. It is shown that when the ratio in the colloidal solution of the amount of the substance $\mathrm{CH}_{3} \mathrm{COOH} / \mathrm{SiO}_{2}=0.43$ is reached, an increase in the polymerization rate is observed, which is the main cause of low aggregative stability. Colloidal solutions containing zinc hydrosilicates synthesized at a concentration of iron (III) hydroxide used to produce a precursor equal to $0.7 \%$ have a longterm aggregative stability and do not significantly change the reduced particle. Such compositions are to be expediently used for the nanomodifying of building composites in order to control their structure formation and to create conditions that impede the development of various mycelial fungi.
\end{abstract}

\section{Introduction}

Currently, one of the priority areas of scientific development is the maintenance of environmental safety, including in the premises of residential buildings and public facilities [1]. This includes both the proper utilization of building materials with the expired service life, and maintenance of favorable environmental conditions in existing buildings and facilities. It is important to ensure a safe microclimate in the premises for the preservation of human health and the specified lifetime of building materials. It is known that mold fungi are

\footnotetext{
*Corresponding author: info@nocnt.ru
} 
more dangerous compared to dust mites, bacteria and animal hair [2-5]. The formation of colonies of mycelial fungi, mainly represented by the genera Penicillium and Aspergillius [2$4,6]$, is often observed on the surface and in the thickness of the enclosing structures or their finishing layers, which are capable of causing various diseases in humans [7,8], since in most are allergenic and conditionally pathogenic; also contribute to the premature destruction of the material $[9,10]$ due to the chemical interaction of the products of the life activity of mold fungi with the components of the building material and with the germination of the mycelium into the thickness of the material [11-13]. Of course, the presence of mold deteriorates the decorative appearance of structures and the appearance of the premises.

The solution of these problems is the creation of modifiers that allow long-term maintenance of the fungicidal properties of the material [14-19] and be safe using [20-23]. One of the fungicidal modifiers having the listed properties is a nanomodifier based on zinc hydrosilicates, which also affects the structure of mineral binders.

However, silica-containing systems, despite the advantages of their use, have features that are their ability to polymerize, and in the presence of metal cations, hetero-coagulation is possible. These processes are accompanied by the coarsening of particles. For the modifiers represented by nanoscale particles, one of the important indicators determining the possibility of its use is the preservation of its aggregative and sedimentation stability during storage. This is necessary for the uniform distribution of the nanomodifier particles in the building material, as well as for the preservation of the efficiency of nanomodification, as assessed by the change in the properties of the resulting composite. Therefore, the purpose of the study was to establish the aggregative stability of a biocidal nanomodifier based on zinc hydrosilicates with the aim of predicting the shelf life of the zinc, allowing use in the construction industry, taking into account the delivery and storage of the nanomodifier before use. The objectives of the study were: establishment of the warranty period for storage of nanomodifier on the basis of zinc hydrosilicates and studying the processes occurring during storage of a colloidal solution of zinc hydrosilicates.

\section{Materials and methods}

The synthesis technology of the nanomodifier, calculation of the concentration of the precursor of silicic acid $(\alpha)$ was previously described in [24] and [25]. According to [26], the fungicidal properties of the material are possible even if the used fungicides have low concentrations that are not qualitatively determined. For nanomodifiers, in addition to the above, a requirement is made to preserve their aggregative stability during storage. The temperature regime during storage was the same for all systems (storage temperature was $20 \ldots 25^{\circ} \mathrm{C}$ ), the compositions were not subjected to stirring, therefore, these factors have an equal effect on all the studied compositions. To estimate the storage time of the nanomodifier based on zinc hydrosilicates, the change in the reduced diameter of its particles after 1 year of storage by the laser diffraction method was studied using a Microtrac Zetatrac apparatus.

\section{Results and Discussion}

The results of studies of the change in the reduced diameter of zinc hydrosilicate particles are shown on figure 1. 


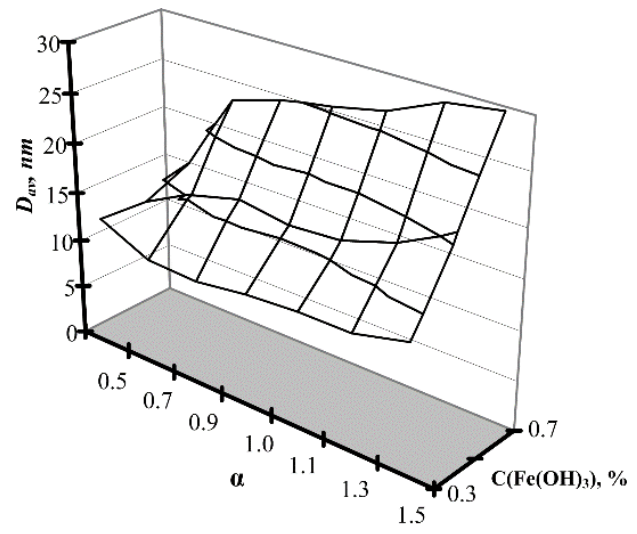

a)

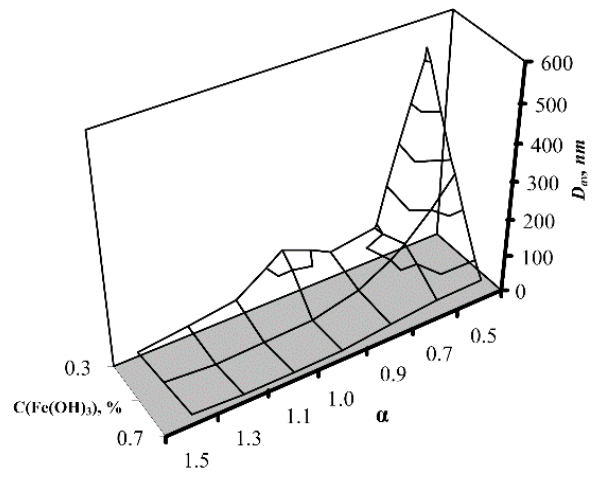

b)

Figure 2. Size of zinc hydrosilicate nanoparticles after synthesis (a) and after 1 year of storage (b).

The analysis of figure 1 shows that the aggregate resistance of synthesized zinc hydrosilicates is influenced by the formulation. Thus, in the composition obtained with the iron (III) hydroxide sol, $0.3 \%$ concentration and the content of the precursor of silicic acid $\alpha$ $=0.5$, a significant increase in particle size is observed - from 10.78 to $526.5 \mathrm{~nm}$. In other compositions, the particle size remains in the nano-range: the particle sizes increase to 130 $\mathrm{nm}$. It is known [27] that in time, depending on the conditions, both polymerization, depolymerization of silica-containing systems, and heterocoagulation are observed. To identify the processes that occur during the storage of colloidal solutions of zinc hydrosilicates, the $\mathrm{pH}$ of the medium has been studied (figure 2).

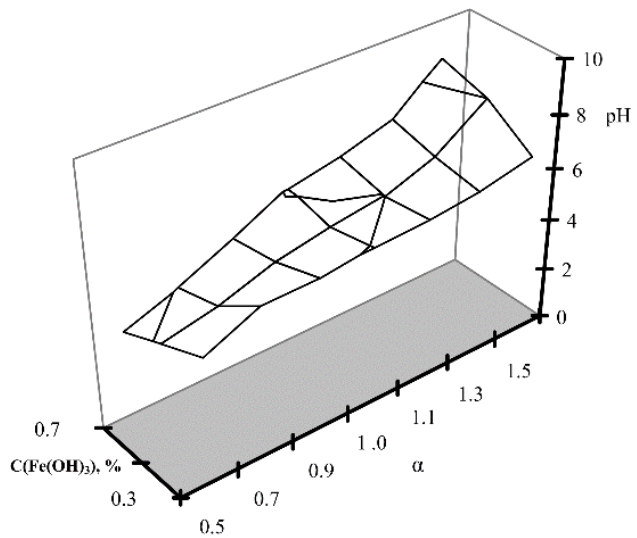

a)

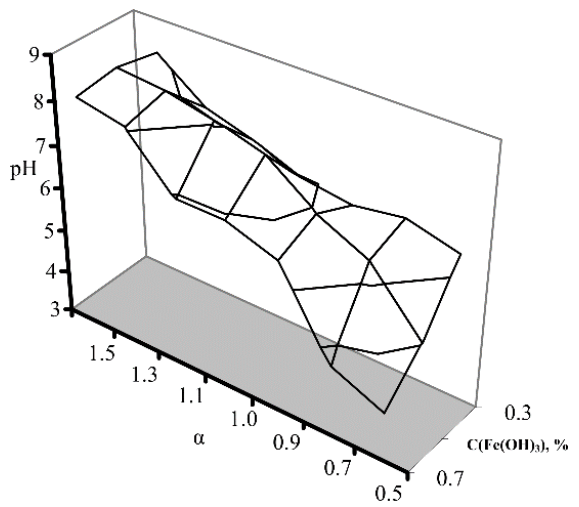

b)

Figure 2. $\mathrm{pH}$ of colloidal solutions of nanosized zinc hydrosilicates after synthesis (a) and after 1 year of storage (b)

It is evident from the data presented in figure 2 that for all compositions an increase in the $\mathrm{pH}$ of the solutions after storage is observed, which indicates the dominance of the polymerization processes. The most significantly increased $\mathrm{pH}$ for compositions synthesized using a sol of iron hydroxide (III), a concentration of $0.3 \%$ and substantially less - for the compositions made using a sol of iron hydroxide (III), a concentration of $0.7 \%$. This is 
consistent with the data of [27], indicating the growth of the $\mathrm{pH}$ of the system due to a decrease in the concentration of acid groups - $\mathrm{SiOH}$.

Also, the concentration of silica and the concentration of the electrolyte, zinc acetate used for the synthesis of zinc hydrosilicates, in particular acetate ions, have a significant effect on the state of colloidal silica-containing systems. The values of the concentration of zinc hydrosilicates in the formulations and the ratio of the amounts of $\mathrm{CH}_{3} \mathrm{COOH} / \mathrm{SiO}_{2}$ are shown in tables 1 and 2 .

Table 1. Concentration of zinc hydrosilicates in colloidal solutions with different synthesis formulas, $\%$

\begin{tabular}{|c|c|c|c|c|c|c|c|}
\hline $\begin{array}{c}\text { Concentration of } \mathrm{Fe}(\mathrm{OH})_{3} \text { sol, } \\
\%\end{array}$ & \multicolumn{7}{|c|}{ Value of $\alpha$} \\
\cline { 2 - 8 } & 0.5 & 0.7 & 0.9 & 1.0 & 1.1 & 1.3 & 1.5 \\
\hline 0.3 & 0.028 & 0.039 & 0.042 & 0.047 & 0.052 & 0.058 & 0.067 \\
\hline 0.5 & 0.041 & 0.057 & 0.066 & 0.073 & 0.080 & 0.091 & 0.105 \\
\hline 0.7 & 0.067 & 0.094 & 0.112 & 0.125 & 0.138 & 0.158 & 0.182 \\
\hline
\end{tabular}

Table 2. Ratio of amounts of $\mathrm{CH}_{3} \mathrm{COOH} / \mathrm{SiO}_{2}$ substance in colloidal solutions for various synthesis formulations

\begin{tabular}{|c|c|c|c|c|c|c|c|}
\hline Concentration of $\mathrm{Fe}(\mathrm{OH})_{3}$ sol, & \multicolumn{7}{|c|}{ Value of $\alpha$} \\
\cline { 2 - 8 }$\%$ & 0.5 & 0.7 & 0.9 & 1.0 & 1.1 & 1.3 & 1.5 \\
\hline 0.3 & 0.028 & 0.039 & 0.042 & 0.047 & 0.052 & 0.058 & 0.067 \\
\hline 0.5 & 0.041 & 0.057 & 0.066 & 0.073 & 0.080 & 0.091 & 0.105 \\
\hline 0.7 & 0.067 & 0.094 & 0.112 & 0.125 & 0.138 & 0.158 & 0.182 \\
\hline
\end{tabular}

It follows from the analysis of the data of Table 1 that the concentration of zinc hydrosilicates in a colloidal solution made with the iron (III) hydroxide sol equal to $0.3 \%$ and at $\alpha=0.5$ is the minimum of those studied. In this case, the aggregative and sedimentation stability of the specified colloidal solution does not allow it to be stored for a long time. This contradicts the known data [27] on the change in the rate of polymerization of silicacontaining colloidal systems, depending on their concentration. However, the ratio of $\mathrm{CH}_{3} \mathrm{COOH} / \mathrm{SiO}_{2}$ in the described composition is the maximum (table 2). According to [28], the influence on the stability of colloidal systems of electrolytes has a contradictory character: it was shown in [27] that with an increase in the concentration of electrolyte, the intensity of its influence on the rate of polymerization of silicic acid is most pronounced in an alkaline medium, and in [29] that electrolytes, depending on the $\mathrm{pH}$ of the medium, can accelerate, slow down or not affect the polymerization rate. Analysis of the data presented in table 2 shows that the relative concentration of acetate ions is the highest in the composition synthesized using the iron (III) hydroxide sol, at a concentration of $0.3 \%$, and at $\alpha=0.5$. Thus, the influence of alkaline medium $(\mathrm{pH}=8.32)$, as well as a high concentration of acetate ions, lead to an increase in the rate of polymerization of zinc hydrosilicates. It is possible to increase the stability of this composition only by decreasing the mass of zinc acetate, which is impractical due to a decrease in the content of zinc, which is the carrier of fungicidal properties. 


\section{Summary}

A technology has been developed for the preparation of a nanomodifier for controlling the structure formation of mineral binders and maintaining the fungicidal properties of building materials. It is shown that the guaranteed shelf life of such a modifier is at least 1 year, except the composition made using the iron (III) hydroxide sol, at a concentration of $0.3 \%$, and at $\alpha$ $=0.5$. The reason for the decrease in the shelf life of the zinc hydrosilicate sol using the iron (III) hydroxide sol, at a concentration of $0.3 \%$ and at a value of $\alpha=0.5$, is an increase in the rate of its polymerization due to the high concentration of electrolyte and alkaline solution medium. It has been established that during the storage of all the investigated colloidal solutions of zinc hydrosilicates, polymerization processes dominate the processes of formation of donor-acceptor bonds between zinc cations and active centers of the surface of silicic.

\section{Acknowledgments}

This work is supported by grant of Russian Federation President MK-8575.2016.8.

\section{References}

1. Technical regulations on safety of buildings and structures: Federal law from 30.12.2009 № 384-FZ (2009)

2. T.M. Zheltikova, A.B. Antropova, E.N. Bilanenko, V.L. Mokeeva, L.N. Chekunova, A.D. Petrova-Nikitina. Pest Management, 1 (2006)

3. O.B. Gradusova, N.E. Ivanushkina, G.A. Kochkina, S.M. Ozerskaja. Theory and practice of forensic examination 3 (2010)

4. O.A. Makanina, L.V. Gordeeva. Bulletin of Belgorod state University. Series: Natural science, 10 (2013)

5. V.M. Katola. Far Eastern journal of infectious pathology, 21 (2012)

6. T.M. Zheltikova. Problems in medical Mycology, 2 (2009)

7. V.S. Lesovoj, A.V. Lipnickij, O.M. Ochkurova. Problems of medical mycology, 2 (2004)

8. T.N. Burkutbaeva, L.K. Tastanbekova. Problems of medical mycology, 3 (2004)

9. V.T. Erofeev et al. Solid State Phenomena, 871 (2016)

10. V.T. Erofeev et al. Procedia Engineering, 165 (2016)

11. L.L. Negoda. Tradition and innovation in construction and architecture. Science and technospheric safety, 4 (2015)

12. V.F. Stroganov, V.A. Bojchuk, E.V. Sagadeev. Proceedings of the Kazan state University of architecture and construction, 2 (2014)

13. S.A. Starcev. Magazine of civil engineering, 7 (2010)

14. H. Wang, X. Xu, Y. Wang, H. Huang, Y. Li. Environmental Toxicology and Chemistry, $3(2011)$

15. F.T. Jesus, R. Oliveira, A. Silva, A.L. Catarino, A.M. Soares, A.J.A. Nogueira, I. Domingues. Ecotoxicology, 9 (2013)

16. J.A. Hingston, A. Bacon, J. Moore, C.D. Collins, R.J. Murphy, J.N. Lester. Bulletin of Environmental Contamination and Toxicology, 1 (2002) 
17. A.R. Fernandez-Alba, M.D. Hernando, L. Piedra, Y. Chisti. Analytica Chimica Acta, 2 (2002)

18. K.E. Appel. Archives of Toxicology, 2 (2000)

19. N. Voulvoulis, M.D. Scrimshaw, J.N. Lester. Chemosphere, 7 (2002)

20. J.O. Morley, A.J. Oliver, M.H. Charlton. Computational and Theoretical Chemistry, 1 (1998)

21. P. Viswanathamurthi, K. Natarajan. Transition Metal Chemistry, 6 (1999)

22. J.A. Ponasik, D.J. Kassab, B. Ganem. Tetrahedron Letters, 34 (1996)

23. M. De Bolle, W.F. Broekaert, B.P.A. Cammue, S. Rees, J. Vanderleyden. Biotechnology Advances, 1 (1997)

24. A.N. Grishina, E.V. Korolev. AIP Conference Proceedings, 1722 (2016)

25. A.N. Grishina. Proc. of XIV International Conference of Students and Young Scientists "Prospects of Fundamental Sciences Development", Tomsk, 6 (2016)

26. V.F. Stroganov, E.V. Sagadeev. Introduction to the bio-construction of building materials. Kazan: KazGASU (2014)

27. N.A. Shabanova, P.D. Sarkisov. Sol-gel technology. Nanodisperse silica. Moscow: BINOM (2012)

28. T. Yokoyama, Y. Takanashi, T. Tarutani. J. Colloid Interface Sci, 2 (1991)

29. S.I Kontorovich., L.N. Sokolova, E.A. Golubeva, E.I. Kochetkova, N.P. Sokolova, E.D. Shhukin. Colloid Journal, 1 (1991) 